\title{
IL CULTO DI SILVANO NEL LIMES DELLA MESIA SUPERIORE*
}

\author{
SANJA PILIPOVIĆ \\ Institute for Balkan Studies \\ Serbian Academy of Sciences and Arts \\ Knez Mihailova 35/IV, 11000 Belgrade, Serbia \\ sanjapil@gmail.com
}

\begin{abstract}
The cult of Silvanus on the limes of Upper Moesia. The subject of the research is the cult of Silvanus in the Danube limes area in the Roman province of Upper Moesia. It has been analyzed eight votive inscriptions of which are preserved only four, and one votive relief related to the cult of this deity. The aim of the research is to elucidate the relationship between Silvanus and agricultural-pastoral world, its indigenous or Italic character and identity of the cult followers. In this work is also stressed the significance of the impact from the neighboring provinces in shaping of the cult, especially from the Lower Pannonia.
\end{abstract}

Keywords: Silvanus, cult, limes, Upper Moesia, Lower Pannonia.

Lo studio quivi presentato propone di esaminare la venerazione del dio Silvano nel territorio del limes danubiano della provincia romana della Mesia Superiore, per mezzo dell'analisi di otto iscrizioni e di un rilievo votivo. Fino ad oggi tali reperti, tuttavia, non sono stati oggetto di un particolare interesse e di studi approfonditi e sono stati esaminati solo indirettamente, trattando altri argomenti. ${ }^{1}$ Si ricorda l'eccellente lavoro di Dorcey (1992) che ne proponeva nella sua monografia dedicata al culto di Silvano nel mondo romano, ${ }^{2}$ un' analisi sommaria in cui confermava le precedenti ricerche di Mócsy, che sosteneva la provenienza del culto in questione dalla Pannonia Inferiore. ${ }^{3}$ Questo è dunque il motivo che ci induce a intraprendere uno studio più approfondito in merito. Prima dell'analisi, si desidera presentare il materiale epigrafico e archeologico nella forma di schede di catalogo. ${ }^{4}$

1. Ara con dedica al dio Silvano

Descrizione: l'ara fu trovata, insieme con altri reperti archeologici riferibili al II-III secolo d.C., nella Fortezza di Belgrado durante gli scavi eseguiti nel periodo 1961-1962, nei resti di una costruzione Ara di materiale o dimensioni non determinabili, irreperibile e perduta. Bibliografia: Kondić 1963, 79; MiRKović-DuŠANIĆ 1976, 57.

* La mia gratitudine va in particolare alla dott. Veselinka Ninković curatrice del Museo Nazionale di Serbia, alla dott. Snežana Cvetković curatrice del Museo Nazionale di Smederevo e alla dott. Dragana Spasić-Đuric curatrice del Museo Nazionale di Požarevac per avermi aiutato nel reperimento delle foto dei pezzi conservati nel loro museo. La mia gratitudine ADD va anche alla prof. Jelena Mrgić, della Facoltà di Filosofia a Belgrado, speciale per aver realizzato la mappa del limes della Mesia Superiore.

${ }^{1}$ Tra i pochi studi si ricordano una tesi di master di VASILJEVIĆ (2008), lo studio sulle paterae (coppe per i sacrifici) provenienti dalla Pannonia e dalla Mesia (Popović 2009, 130), lo studio sulle dediche alle divinità silvestri nei Balcani (PLEMIĆ 2012, 48, 51) ed una tesi di dottorato sulla produzione e importazione del grano nella Mesia Superiore (ŽIVANOvić 2013, 102-105).

2 DORCEY 1992, 75.

${ }^{3}$ Mócsy 1970, 244

${ }^{4}$ È importante notare i due monumenti, che non fanno parte del catalogo: l'ara votiva (IMS II, 41) dedicata alle Deae Silvestres, proveniente da Viminacium ed un rilievo, oggi irreperibile, di provenienza sconosciuta che era in possesso del Museo Nazionale di Serbia (VULIĆ-LADEK-PREMETŠTAJN 1931, n. 619). Il rilievo raffigurava le gambe di Pan, una grande anfora e due ninfe che ballano tenendosi per mano. Questi non fanno parte del catalogo perché nel primo caso non si tratta di una dedica al dio Silvano e nel secondo perchè la provenienza del rilievo rimane sconosciuta. 
2. Ara con dedica al dio Silvano (fig. 1)

Descrizione: Ara votiva di calcare, con forma di un parallelepipedo a base rettangolare e zoccolo circondato da una doppia modanatura. La superficie superiore è allargata e marcata da una cornice. La fronte è occupata dallo specchio epigrafico. Manca la parte superiore sinistra dell'ara.

Misure: $\quad 47 \times 24 \times 25 \mathrm{~cm}$

Provenienza: $\quad$ Vračar, municipio di Belgrado.

Datazione: $\quad$ I-III secolo d.C.

Collocazione: $\quad$ Museo Nazionale di Serbia, collezione dei monumenti epigrafici antichi e medievali, inv. 69.

Iscrizione:

Silvano | Silvestr $[i]$ | Iul(ius) Se(p)tu| mus v(otum) p(osuit).

Bibliografia: $\quad$ Vulić 1931, 7, n. 7; Iscrizione: CIL III, 6304; IMS I, 22.

3. Ara con la dedica a Silvano (fig. 2)

Descrizione: Ara votiva di arenaria giallastra, con forma di parallelepipedo a base rettangolare, e zoccolo modanato. La superficie superiore è allargata e marcata da una cornice. La fronte è occupata dallo specchio epigrafico. Leggermente danneggiata, specialmente alla base e alla cornice.

Misure:

$39 \times 26 \times 12 \mathrm{~cm}$

Provenienza: Guberevac, municipio di Stojnik, montagna di Kosmaj.

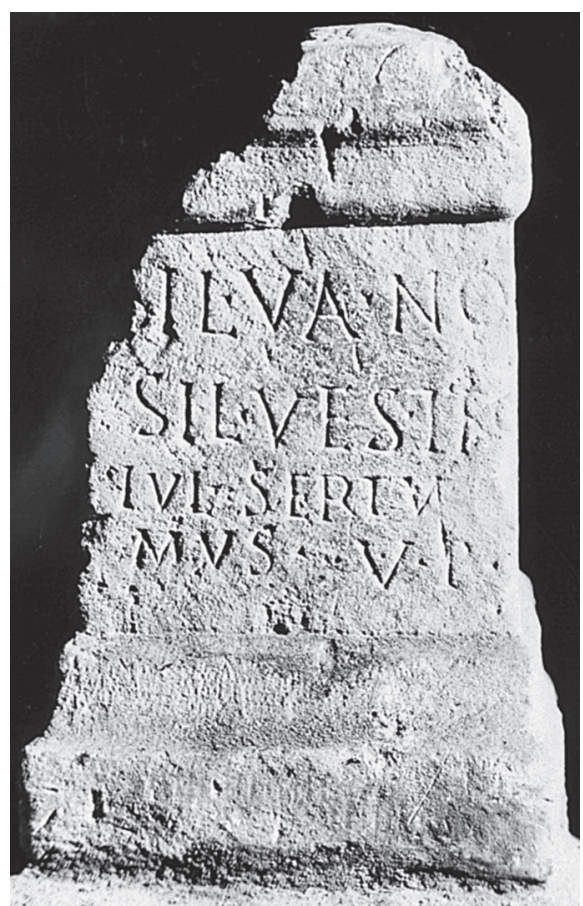

Fig. 1. Ara votiva dedicata Silvano proveniente da Vračar, Belgrado (IMS I 22). Museo Nazionale di Serbia, inv. 69. Foto: Museo Nazionale di Serbia, Belgrado

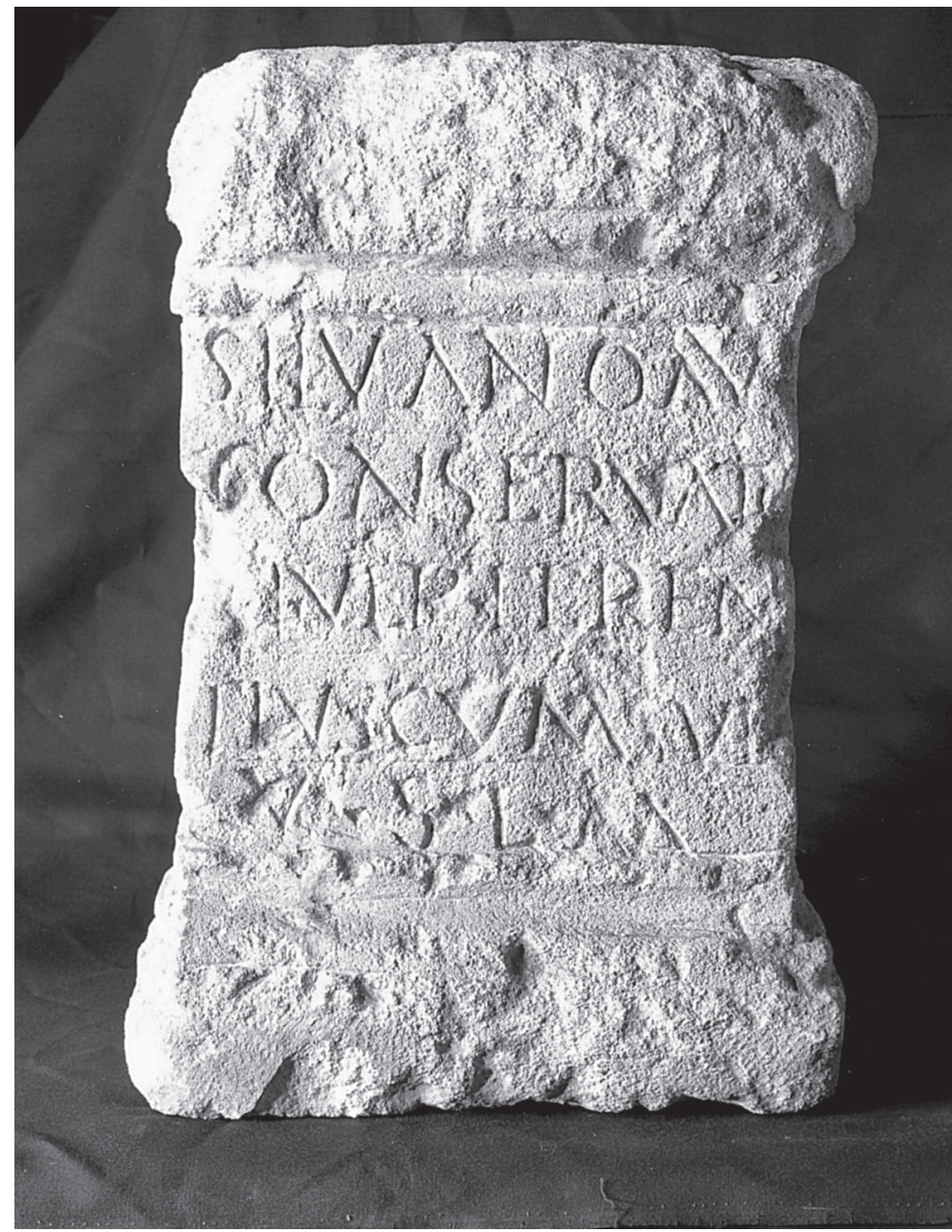

Fig. 2. Ara votiva dedicata a Silvano da Guberevac, Kosmaj (IMS I 108). Museo Nazionale di Serbia, inv. 5. Foto: Museo Nazionale di Serbia, Belgrado 
Datazione: I-III secolo d.C.

Collocazione: Museo Nazionale di Serbia, collezione dei monumenti epigrafici antichi e medievali, inv. 5.

Iscrizione:

Silvano Au(gusto) | Conservato(ri) | M. Ulp(ius) Teren I tius cum sui [s] I v(otum) s(olvit) l(ibens) m(erito).

Bibliografia: $\quad$ MATIĆ 1955, 358, n. 1, fig. 4. Iscrizione: ILJug 25; IMS I, 108.

4. Ara con la dedica a Silvano

Descrizione: Ara votiva con forma di parallelepipedo a base rettangolare, e zoccolo modanato. La superficie superiore è allargata e marcata da una cornice. Il lato posteriore e il lato destro sono grezzi. La fronte è occupata dallo specchio epigrafico. Danneggiata, specialmente alla base e alla cornice.

Misure: $\quad 65 \times 30 \times 25 \mathrm{~cm}$

Provenienza: $\quad$ Sibnica, nel municipio di Sopot, montagna di Kosmaj. L'ara è stata trovata vicino all'apside di una costruzione più antica.

Datazione: I-III secolo d.C.

Collocazione: $\quad$ Davanti alla chiesa di Santa Parascheva nel Monastero di Sibnica.

Iscrizione:

Silvano D(omestico) | Oppius | Maximus | $v$ | otum s(olvit) l(ibens) $m$ | erito

Bibliografia: $\quad$ JovanOVIĆ-PoP-LAZIĆ-MrKoBRAD 1992, 139-140

5. Ara con la dedica a Silvano

Descrizione: L'ara proviene dal sito di Grad, nel municipio di Stojnik, montagna di Kosmaj. Ara di materiale e dimensioni non determinabili, ai nostri tempi irreperibile. Esiste solo una notizia da Vulić (1913, 344, fig. 2) che menziona che l'ara era stata dedicata a Silvano da un beneficiarius.

Bibliografia: $\quad$ VuLIĆ 1913, 344, fig. 2; Iscrizione: IMS I, 109

6. Ara con dedica a Silvano

Descrizione: Ara votiva di calcare con forma di parallelepipedo a base rettangolare, e zoccolo a doppia mondatura. Il coronamento dell'ara, abbastanza rovinato, è composto di un acroterio centrale racchiuso da due acroteri laterali. La fronte è occupata dallo specchio epigrafico. Danneggiata specialmente alla base e alla cornice. L'ara è citata dalle fonti bibliografiche ma non è rintracciabile.

Misure: $\quad 56 \times 24 \times 12 \mathrm{~cm}$

Provenienza: Smederevo.

Datazione: $\quad$ II-III secolo d.C.

Iscrizione:

Silva(no) | Dom(estico) | EGNIM

Bibliografia: $\quad$ VuLIĆ 1931, 242, n. 645; Iscrizione: IMS II, 310.

7. Ara con dedica a Silvano e Diana (fig. 3)

Descrizione: Ara votiva di calcare con forma di parallelepipedo a base rettangolare, e zoccolo a doppia mondatura. Il coronamento dell'ara è abbastanza rovinato ed è decorato con i due acroteri laterali. La fronte è occupata dallo specchio epigrafico. Danneggiata specialmente alla base e alla cornice.

Misure: $\quad 56 \times 25 \times 25 \mathrm{~cm}$

Provenienza: Kalište, a sud di Kostolac.

Datazione: $\quad$ Dalla seconda metà del II al III secolo d.C.

Collocazione: $\quad$ Museo Nazionale di Požarevac, inv. II/2681.

Iscrizione:

IDAVNI ? | Apollin(i) | Q? S Dian | a(e) Silvano | Ael(ius) Vale | ntinus | IIvir col(oniae) | v(otum) l(ibens) p(osuit)

Bibliografia: $\quad$ CLAuss 1988, 159-160; BREIN 1966-1967, 79-86; Iscrizione: ILJug 488; IMS II, 297 ; AEp $1988,980$.

8. Ara con dedica a Silvano

Descrizione: Ara votiva di arenaria con forma di parallelepipedo base rettangolare, e zoccolo a doppia mondatura. Il coronamento dell'ara è abbastanza rovinato ed è decorato con una vite sita tra i due acroteri laterali. La fronte è occupata dallo specchio epigrafico. Danneggiata specialmente alla cornice. L'ara citata dalle fonti bibliografiche non è però rintracciabile.

Misure: $\quad 64 \times 39 \times 33 \mathrm{~cm}$

Provenienza: Kalište, a sud di Kostolac.

Datazione: II-III secolo d.C 


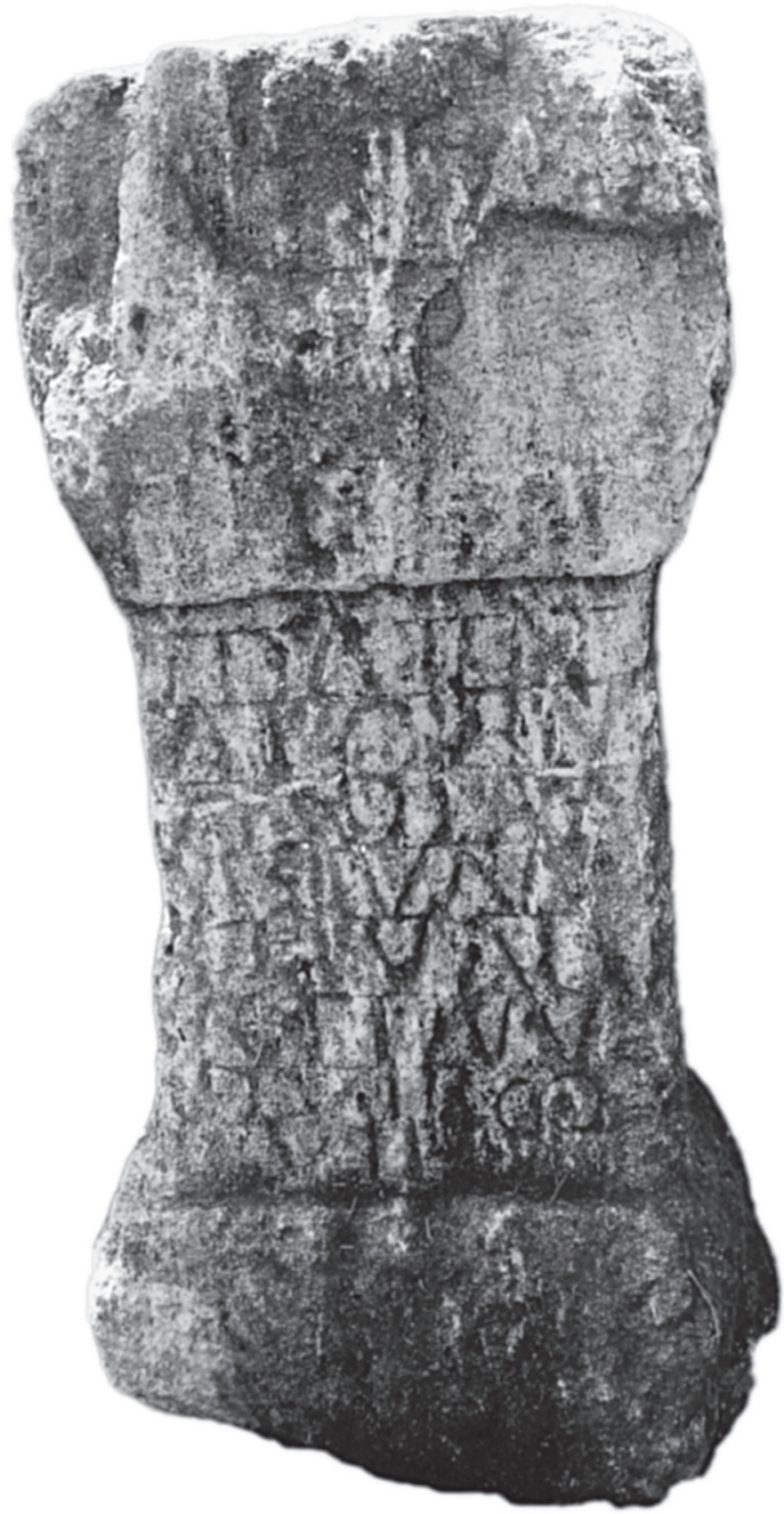

Fig. 3. Ara votiva dedicata a Silvano da Kalište (IMS II 297). Museo Nazionale di Požarevac, inv. II/2681. Foto: Museo Nazionale di Požarevac 
Iscrizione:

Sil(vano) I sac(rum) I Dom(estico)

Bibliografia: $\quad$ Vulić 1909a, 143, n. 65; VUlić 1909b, 171-172, n. 34; fig. 120; Iscrizione: AEp 1910, 92 ; IMS II, 300.

9. Rilievo murato nella fortezza di Smederevo (fig. 4, fig. 5)

Descrizione: Rilievo di calcare grigiastro. Tre figure maschili in piedi una accanto all'altra. Nella parte sinistra si riconosce una figura barbata, vestita di tunica, con berretto frigio in testa. Nella mano destra regge una falce (falx alboraria, falx putatoria) e nella mano sinistra un ramo di pino. Al centro si vede una figura nuda che nella mano sinistra regge uno scettro (sceptrum). Nella parte destra vi è una figura nuda maschile che regge qualcosa in mano, ma il rilievo è poco chiaro. Composizione armoniosa. Provenienza: Kalište, a sud di Kostolac.

Datazione: $\quad$ Seconda metà del II secolo d.C.

Collocazione: $\quad$ Fortezza medievale a Smederevo. Il rilievo è murato nella prima torre del muro orientale, in alto, quasi in cima. Bibliografia: $\quad$ Vulić-LAdEK-Premetštajn 1931, 66; ; CVETKović 2009, 35-36; ; CVetKović 2011, 401-402.

Il territorio del limes, il bacino a sud del Danubio, date le sue caratteristiche particolari, si presenta come un'entità separata della Mesia Superiore. Si tratta di un bassopiano a sud della Pianura Pannonica, con i bacini dei fiumi Danubio (Danuvius), Grande Morava (Margus), Timok (Timacus) ed altri, caratterizzati da terra alluvionale, coltivabile lungo gli argini. ${ }^{5}$ Era una nota base militare già dalla fine del I secolo d.C., dal tempo della guerra con i Daci ed ospitava l'esercito romano spostato lungo il Danubio. ${ }^{6}$ In seguito, durante i secoli della dominazione romana questo territorio non ha mai perduto la sua rilevanza. Il territorio possedeva eccellenti vie di comunicazione, sia terrestri che marittime. Già dall'inizio del I secolo le vie situate nella terra ferma erano importanti e vi si trovavano strutture ausiliarie per l'utilizzo dei fiumi navigabili. ${ }^{7}$

Sembra che questo territorio di carattere agricolo, lontano dai centri cittadini del limes, composto da boschi e terra coltivabile fosse favorevole allo sviluppo del culto di Silvano. Si ha notizia di un'unica ara votiva, oggi ormai perduta, che era stata trovata nel castrum di Singidunum (cat. 1). Invece, le altre dediche non provengono dai centri urbani oppure non hanno un'esatta indicazione di provenienza. Infatti, un'altra dedica proviene invece dalla periferia del territorio di Singidunum (cat. 2). Due dediche provengono dal castellum vicino a Kalište (cat. 7 e 8), che si trovava sulle rive del fiume Vitovnica, $26 \mathrm{~km}$ a sud di Kostolac (Viminacium). ${ }^{8}$ Tre dediche provengono dalla zona della miniera della montagna di Kosmaj (cat. 3-5), ${ }^{9} 40 \mathrm{~km}$ a sud di Belgrado, due dal municipio di Stojnik e una dal municipio di Sopot. Una dedica, oggi ormai irreperibile, proveniente da Smederevo (cat. 6) e il rilievo (cat. 9) murato nella fortezza medievale di Smederevo, non hanno un'esatta indicazione di provenienza. Certi studi ipotizzano che i monumenti murati in questa fortezza possano provenire da Viminacium, Margum oppure Smederevo, cioè Vinceia e Aureus Mons. ${ }^{10}$ Secondo Vulić, questo rilievo proviene da Viminacium. ${ }^{11}$ Pertanto, non siamo in grado di indicare una datazione precisa dei monumenti citati. Essi possono risalire a un periodo dal I secolo fino alla fine del III (cat. 2, 3, 6, 8), o al II o III secolo (cat. 4), o più precisamente alla seconda metà del II secolo (cat. 9).

Prima di proseguire con l'analisi, è opportuno notare le caratteristiche di Silvano in Italia, paese di origine di questo culto. ${ }^{12}$ Silvano fu un dio popolare che, tutelava il mondo rurale-pastorale e garantiva il benessere a quanti svolgevano la loro attività in aziende agricole o in pascoli. La sua protezione si estendeva poi alla domus, a cui garantiva sicurezza, buona condizione fisica e prosperità. Col tempo la protezione della divinità fu indirizzata anche ai bisogni urbani, quindi, non più ai soli coltivatori e cacciatori, ma anche alla gente semplice delle città.

\footnotetext{
${ }^{5}$ Si tratta di buona terra alluvionale lungo i fiumi, ILIĆ 2012, 22 con ampia bibliografia precedente.

${ }^{6}$ Mirković 1968, 22-35

${ }^{7}$ La via danubiana che, seguiva il corso del Danubio lungo l'intero limes fino alla sua foce, congiungeva le città romane e le fortificazioni militari sorte lungo il fiume. Il Danubio non era l'unico fiume navigabile, c'erano anche i fiumi Sava (Savus) e Grande Morava (Margus). Per i porti lungo il Danubio e Classis Flavia Moesica si veda Petrović 1991, 207-216; DimitriJević 1996, 143-157. Esisteva una rete di strade terrestri importanti ben collegate tra loro. La strada da Singidunum verso Serdica e Constantopolis, la strada da Singidunum e Viminacium verso Ratiaria collegavano gli insediamenti e le fortezze della Mesia Superiore lungo la riva destra del Da-
}

nubio, mentre da Ratiaria la strada portava anche verso sud e Naissus, attraverso la valle di Timok; v. Petrović 2009, 139 sqq, 155 sqq; Petrović 2011, 404-414.

${ }^{8}$ Castellum vicino a Kalište, fu costruito dalla legione VII Claudia, Mirković 1968, 68-69; Mirković 1986, 56

${ }^{9}$ DUŠANIĆ $2004,247-270$ con precedente bibliografia.

${ }^{10}$ Cvetrović 2009, 30 sq, con precedente bibliografia

${ }^{11}$ Vulić-LAdEK-Premetštajn 1931, 66.

${ }^{12}$ Per il profilo del dio si veda Peter (1909-1915, coll. 824-877), SALETTI (1966, 297); NAGY (1994, 763-773) con panorama completo sull'iconografia della divinità e la bibliografia, e uno studio approfondito di DORCEY (1992). 
Silvano è una divinità antica, che si ricollega alle radici del popolo romano e la sua flessibilità consentiva la sopravvivenza del culto. Le caratteristiche principali del dio Silvano italico si notano nella letteratura romana. Orazio (Odi, III, 29, 22-23) lo chiama horridi dumeta Silvani o tutore finium (Epod. 1, 22), mentre per Virgilio è arvorum pecorisque deo (Aen. VIII, 600). I gromatici veteres (Scriptores gromatici veteres, I, 302), ci rivelano i tre aspetti del dio: domesticus - quello in cui aveva come campo d'azione la casa; agrestis - quello indirizzato alla natura ed ai pascoli, e orientalis - cioè colui che manteneva e proteggeva i confini. La sua doppia natura di divinità delle

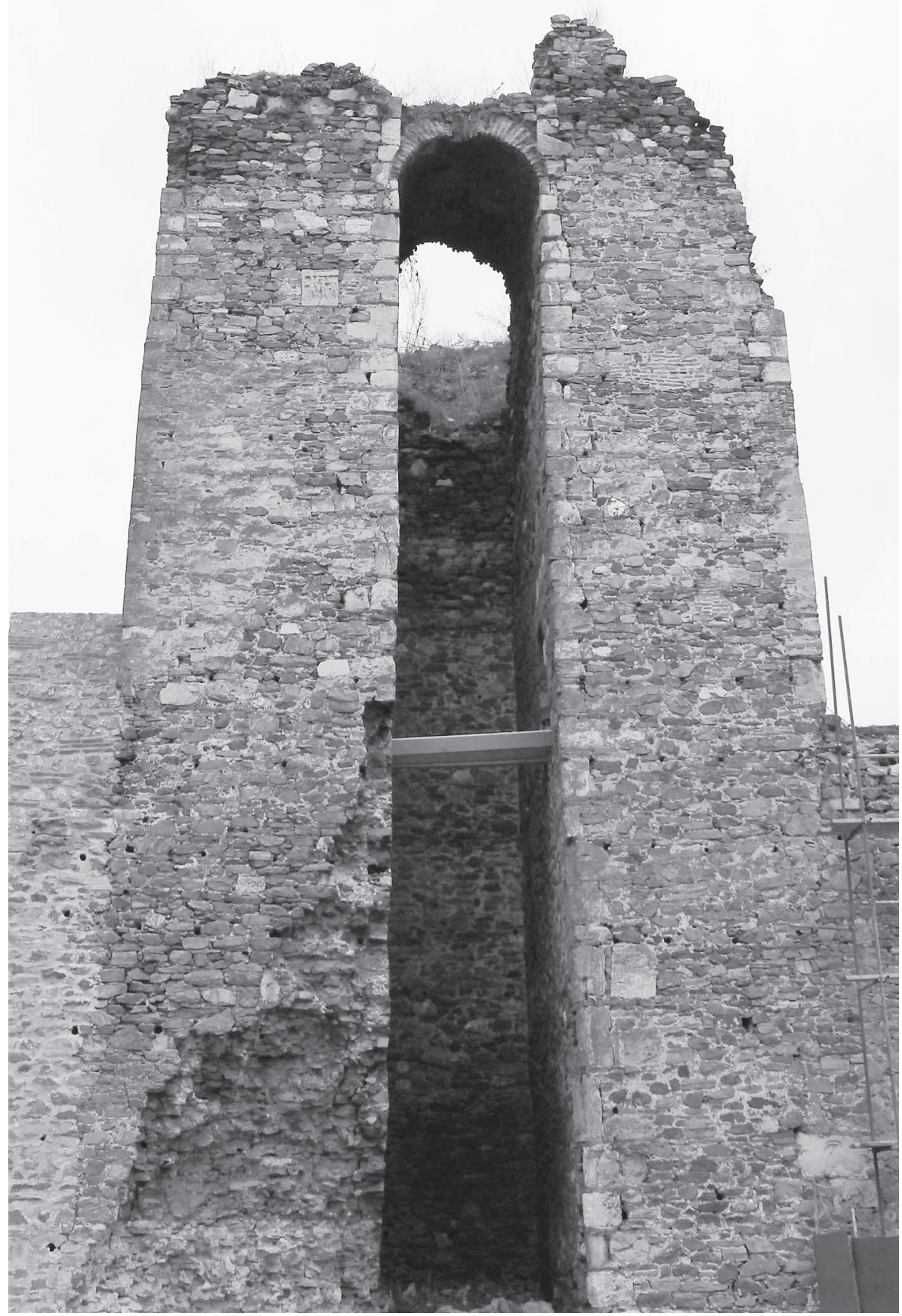

Fig. 4. Torre della fortezza medievale di Smederevo con rilievo murato. Foto: Andrija Pajić 


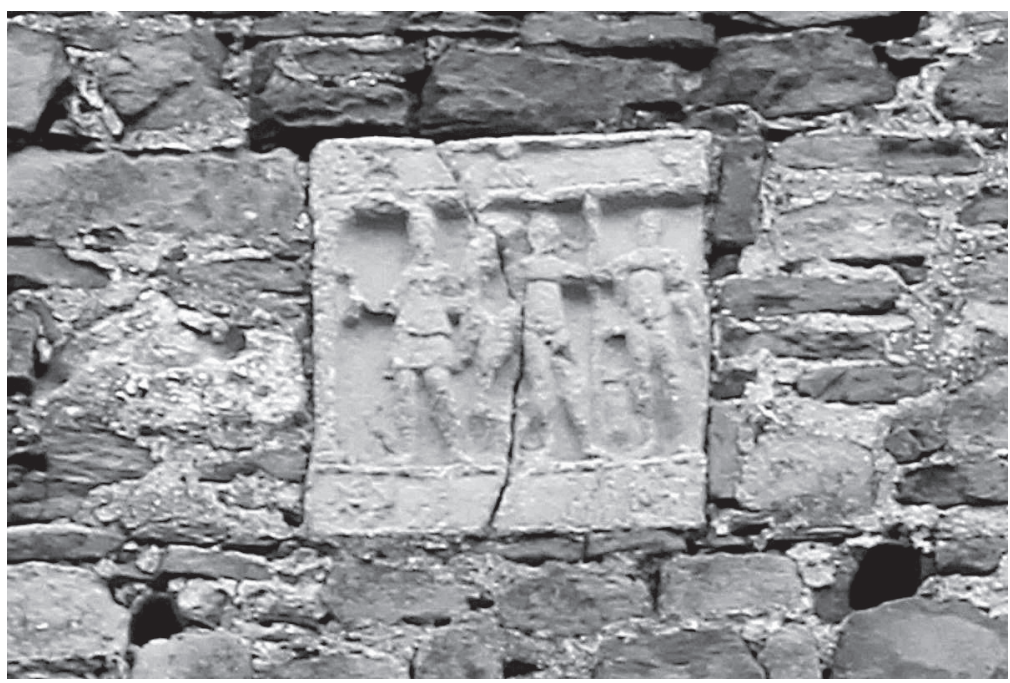

Fig. 5. Rilievo votivo con Silvano murato nella fortezza medievale di Smederevo. Foto Andrija Pajić

foreste e del paesaggio coltivato si riflette nell'iconografia e nell'epigrafia. Di solito Silvano si rappresentava vestito o nudo, con un cono pieno di frutta, oppure con la falce e il pino sotto forma di un albero o un ramo ed un cane accanto. ${ }^{13}$ La maggior parte delle dediche a Silvano attribuisce a tale divinità l'appellativo di Sanctus, e poi ci sono appellativi legati alla vita rurale come silvester, agrestis, incultus, oppure in senso di protezione come custos, conservator, casanicus, oppure appellativi del tutto inaspettati per una divinità rurale come ad esempio coelestis, felix, paesus, geminus. ${ }^{14}$

Il culto di Silvano, di questa divinità italica fioriva nelle province romane dei Balcani in modo multiforme, sia come la venerazione della divinità romana, sia come la cd. interpretatio romana, processo questo con cui la divinità romana veniva identificata nei suoi equivalenti nativi. ${ }^{15}$ Nella Pannonia si venerava Silvano sotto il suo aspetto italico, probabilmente sotto l'influenza esercitata dalla conquista romana di questo territorio e dalla presenza delle truppe romane. ${ }^{16}$ Prevaleva la denominazione di Silvanus Domesticus, mentre l'appellativo Silvester appariva solo in un quarto di tutte le dediche a Silvano. ${ }^{17}$ Gran parte delle dediche al dio Silvano ritrovate nella Pannonia è datata al periodo di Settimio Severo, in cui si osserva la fioritura della proprietà privata. ${ }^{18}$ Probabilmente Silvano non era solo il protettore delle case e delle proprietà site nel territorio suburbano degli insediamenti, ma, in queste zone frontaliere dell'Impero veniva ritenuto anche tutor finium. ${ }^{19}$ Silvano era una divinità molto popolare anche nella Dacia, con il prevalente appellativo di Domesticus e veniva rappresentato con aspetto italico. ${ }^{20}$ Pur essendo stata la sua origine oggetto di ipotesi e tesi diverse, tuttavia è evidente l'analogia con il suo culto sorto in Pannonia. ${ }^{21}$ Nella Dalmazia, al contrario della Pannonia e della Dacia, prevaleva l'appellativo di Silvester con un aspetto teriomorfo della divinità. Poche erano le dediche a Silvano Domesticus, anzi, per quanto si sappia, vi è solo una dedica. ${ }^{22}$ La critica ha ipotizzato che uno dei più diffusi culti di Silvano, quello praticato in Dalmazia, fosse la continuazione di un culto indigeno illirico di età preromana e che attraverso la interpretatio romana fosse stato assimilato alla

${ }^{13}$ DorCeY 1992, 17sqq.

${ }^{14}$ DoRCEY 1992, 29-32

${ }^{15}$ DORCEY 1992, 40.

${ }^{16}$ L'ipotesi che nella Pannonia esistesse una sintesi de culto italico e illirico, cioè pannonico, è stata negata e gli ultimi studi dimostrano che la figura di Silvano nella Pannonia era un riflesso del Silvano italico. Il gran numero di dediche può essere una delle ragioni più importanti per concludere che si tratta di una divinità indigena pannonica, ma non sono presenti molte caratteristiche locali, MócsY 1974, 250; Dorcey 1992, 71; Perinić Muratović-Vulić 2009, 165-180.

${ }^{17}$ Un gran numero di iscrizioni, secondo solo a quelle a Giove, era dedicato con quest'appellativo proprio a Silvano, PERINIĆ
Muratović 2009, 244, 300; Perinić Muratović-Vulić 2009, 166, $169,176,178$.

${ }^{18}$ Fitz 1993, 420-421; Perinić Muratović-Vulić 2009,

176.

${ }^{19}$ Perinić Muratović-Vulić 2009, 178.

${ }^{20}$ DORCEY 1992, 76;

${ }^{21}$ Dorcey 1992, 77 sqq.

${ }^{22}$ In gran parte delle dediche a Silvano trovate in Dalmazia sono prive di qualsiasi appellativo, altre contengono l'appellativo $\mathrm{Au}$ gustus e Silvestris, e infine c'è una a Silvanus Domesticus (CIL III, 14985). 
divinità italica di Silvano. ${ }^{23}$ Nella Mesia Inferiore, il suo carattere è più complesso e sembra che il sincretismo con le divinità indigene fosse più forte. ${ }^{24}$

Per comprendere meglio le caratteristiche del culto di Silvano nel limes della Mesia Superiore è importante innanzi tutto esaminare i dati che ci offrono le analisi epigrafiche e iconografiche. Conosciamo sei dediche in cui il nome del dio è accompagnato da diversi appellativi, e in tre di queste dediche appare l'appellativo Domesticus (cat. $4,6,8)$. Quest'appellativo certifica che Silvano fu venerato in queste zone come protettore del focolare domestico, della casa stessa e di tutto ciò che ad essa si collega, e come protettore della terra coltivabile. ${ }^{25}$ Nella Mesia Superiore l'appellativo Domesticus, oltre ad accompagnare Silvano, appare solo una volta. Da Viminacium proviene una dedica a Giove, agli Dei, alle Dee, ed ai Lari domestici ${ }^{26}$ con i quali si potrebbe paragonare anche Silvano quale protettore del focolare domestico. ${ }^{27}$ Nella dedica da Kalište (cat. 8), Silvano è menzionato con due appellativi Domesticus e Sacrum. ${ }^{28}$ Silvano Domesticus era molto popolare nella Pannonia, specialmente negli insediamenti civili accanto ai campi militari lungo il Danubio, dove si era sviluppata l'agricoltura, ${ }^{29} \mathrm{e}$ anche nella Dacia dove l'appellativo usato più spesso era Domesticus. ${ }^{30}$

Dal limes della Mesia Superiore proviene solo una dedica a Silvano con l'epiteto Silvester (cat. 2) dalla periferia di Singidunum. L'appellativo Silvester riguardava l'uomo che svolgeva attività fuori di casa, incluso la coltivazione della campagna, la protezione delle foreste e dei boschi e la protezione dei pascoli e del bestiame. ${ }^{31}$ L'appellativo Silvester, evidentemente mette in rilievo il legame con il mondo delle silvae, già implicito nel teonimo. È importante notare che nella regione dei Balcani centrali le dediche a Silvanus Silvester sono più frequenti che altrove ${ }^{32}$ e che quest'appellativo nella Dalmazia prevaleva con un aspetto teriomorfo della divinità. ${ }^{33}$

Oltre all'ara votiva proveniente dalla periferia di Signidunum (cat. 2), nel limes della Mesia Superiore fu trovato solo un unico appellativo Silvester, che proviene da Viminacium, ${ }^{34} \mathrm{Si}$ tratta di una dedica alle Deae Silvestres, cioè alle ninfe Nymphae Silvestres,$^{35}$ datata alla seconda metà del II o al III secolo d.C. Queste ninfe, Nymphae Silvestres, erano legate a Silvano. ${ }^{36}$ In base al nome del dedicante, Achilleus, si potrebbe pensare che si tratti di un devoto di origini greche e sottintendere una sua originaria condizione servile. ${ }^{37}$ Questa dedica alle Ninfe è un'importante testimonianza, infatti ci rivela che, le divinità silvestri erano venerate non solo nella periferia di Signidunum (cat. 2), ma anche a Viminacium.

La dedica a Silvanus Augustus Conservator è stata ritrovata solo una volta (cat. 3) nella montagna di Kosmaj. ${ }^{38}$ L'appellativo di Augustus venne attribuito a Silvano a partire dall'età Flavia e Antonina, e divenne corrente

\footnotetext{
${ }^{23}$ Questa interpretazione vede la luce nei primi studi su quest'argomento effettuati intorno all'inizio e nella prima metà del XX secolo (HiRsCHFElD-SCHNeIDER 1885, 34-47; DomASZEWSKI 1902, 1-25) fino agli studi più recenti (RENDIĆ-MiočEvić 1989, 461506; Rendić-MiočEvić 2007a, 33-43; Rendić-MiočEvić, 2007b, 13-30; MatiJašić-TassauX 2000, 76-91; Perinić Muratović-Vulić 2009, 165-180; BEKAVAC, 2011, 151-166; DZino 2012, 261-279). D'altra parte, ci sono alcune ricerche che negano l'esistenza di elementi indigeni nel culto di Silvano nella Dalmazia, Mócsy 1974, 250-252; NAGY 1994, 773; DORCEY 1992, 68-71.

${ }^{24}$ Tra le sedici dediche al dio Silvano, sia Domesticus sia Silvestris, ci sono due dediche a Silvano et Silvestri (AEp 1955, 67; CIL III, 12367). In questi casi si può pensare a a due divinità diverse e che Silvestris non fosse solo un'aggettivazione di Silvano, ma una divinità locale indipendente. Una di queste dediche si trova nel rilievo del Cavaliere tracio e ci rivela un sincretismo con questa divinità tracia, DoRCEY 1992, 76.

${ }^{25}$ Rendić-Miočević 1980, 111-113. DORCEY 1992, 22sqq.

${ }^{26}$ IMS II, 24: I(ovi) O(ptimo) M(aximo) I dis deabusq(ue) I et Larib(us) domest(icis) | L(ucius) Dom(itius) Anthi[--] I po[--

${ }^{27}$ Silvano Domesticus è paragonabile ai Lari del focolare domestico, RENDIĆ-MiočEvić 1980, 111-113.

${ }^{28}$ Silvanus Sacrum è attestato due volte nel sud della Mesia Superiore, a Ulpiana (ILJug 526; ILJug 533). L'appellativo di Silvano Sacrum era molto diffuso nella Dalmazia, dove spesso si trovano uniti gli appellativi di Sacrum e di Silvester, mentre nella Panno-
}

nia e nella Dacia troviamo uniti più frequentemente gli appellativi di Sacrum e di Domesticus, secondo EDH, http://edh-www.adw.uni-heidelberg.de/, (consultato 3.6.2016).

${ }^{29}$ Perinić Muratović-Vulić 2009, 178.

${ }^{30}$ Silvano veniva invocato col titolo di Domesticus nella Dacia in sessanta dediche, mentre l'appellativo Silvester appare solo sette volte, secondo EDH, http://edh-www.adw.uni-heidelberg.de/, (consultato 3.6.2016).

${ }^{31}$ Silvanus Silvester, come Pan, è custode del bestiame, in particolare quello dei piccoli animali, come lo rappresenta Virgilio (Georg. I, 16) chiamandolo "Pan, ovium custos", RENDIĆ-MiočEviĆ 1980, 112, fut. 41; Zotović 1992, 102-104; JovanoviĆ 2000, 19-25.

${ }^{32}$ Dorcey 1992, 18.

${ }^{33}$ ReNDić-MiočEvić 1980, 112 sqq.

${ }^{34}$ IMS II, 41: Deabus silv | estris (!) | Achi | leus ex $v \mid$ oto l(ibens) pos(uit).

${ }^{35}$ MARIĆ 1933, 60.

${ }^{36}$ RENDIĆ-MiočEvić 1989, 483.

${ }^{37}$ Mirković 1986, 80, n. 41.

${ }^{38}$ Accanto alla dedica a Silvano (cat. 3), l'appellativo $\mathrm{Au}$ gustus nel limes della Mesia Superiore appare tre volte assieme a Cerere (IMS II, 3; IMS II, 4; CIL III, 8085), due volte unito a Hercules (AEp 1989, 631; IMS II, 11), una volta a Giove (IMS I, 91), una volta a Nemesis (IMS I, 19), una volta a Sedatus (CIL III, 8086) e una volta assieme a Liber (IMS II, 27). 
ed usuale nella religione ufficiale nell'età dei Severi. ${ }^{39}$ Questo appellativo di Silvano, come tante volte succede nei casi di dediche a Marte e Saturno con appellativi Augustus, si interpreta difficilmente. Probabilmente si tratta di un posto assicurato alla divinità locale nel pantheon romano. ${ }^{40}$ In questa maniera la gente, specialmente la popolazione di origini umili, tentava di plasmare il proprio sistema religioso in modo da collegarsi più strettamente alle divinità e anche agli Imperatori romani. È difficile che l'appellativo Augustus si collegasse al culto ufficiale, poiché si trattava di un culto privato. Come le ricerche riguardanti il culto di Silvano nel territorio circostante hanno già dimostrato, sono pochissimi gli altari votivi, dove Silvano è invocato insieme alla Triade Capitolina oppure a Giove Ottimo Massimo. ${ }^{41}$ Inoltre, le dediche a Silvanus Augustus provengono da santuari privati, lontani dal centro delle città, eretti da dedicanti di umili origini, e giungono da luoghi in cui la romanizzazione era più forte. ${ }^{42}$ Per quel che riguarda le provincie circostanti si osserva che, le dediche a Silvano Augustus erano molto frequenti nella Dalma$\mathrm{zia}^{43}$ al contrario della Pannonia, dove sono state trovate solo quattro ${ }^{44}$ e della Dacia dove sono state reperite solo due dediche. ${ }^{45}$

Nella stessa dedica di Kosmaj Silvano veniva invocato col titolo di Conservator (cat. 3) che sta ad indicare che era ritenuto il "conservatore", cioè il custode, o guardiano, destinato a una protezione in senso lato. ${ }^{46}$ Conservator non era un appellativo comune di Silvano nella Dalmazia, ${ }^{47}$ e neanche nella Pannonia. ${ }^{48}$ D'altra parte, nei Balcani, accanto a Silvano, diverse divinità venivano invocate col titolo di Conservator, come Giove, Ercole, Apollo, Nettuno ect. ${ }^{49}$ Dal limes della Mesia Superiore provengono tre dediche con quest'appellativo, tutte da Viminacium: due a Giove ${ }^{50} \mathrm{e}$ una a Nettuno. ${ }^{51}$ Esiste anche un'interpretazione, difficile da verificare, che ipotizza che in questa dedica, proveniente da Kosmaj (cat. 3) Silvano Conservator avesse la funzione di proteggere le miniere. ${ }^{52}$ Questa tesi si basa sul fatto che Silvano era il patrono di tutte le risorse naturali e cosi anche delle miniere situate in zone montagnose.

Nel limes della Mesia Superiore troviamo due volte il dio Silvano associato al culto di altre divinità. La dedica a Silvano, Apollo, Diana ed un'altra divinità, purtroppo di identificazione incerta, rinvenuta a Kalište (cat. 7), ${ }^{53}$ rende evidente la complementarietà di questi culti, negando così l'ipotesi di una sovrapposizione e assimilazione. Sostanzialmente Silvano e Diana spuntano spesso nelle iscrizioni ritrovate nei Balcani e simili dediche provengono anche dalla Dalmazia, ${ }^{54}$ dalla Mesia Inferiore, ${ }^{55}$ dalla Dacia ${ }^{56}$ e solo un'unica anche dalla Pannonia Inferiore. ${ }^{57}$ Dal punto di vista iconografico, nella Dalmazia si rappresentava spesso Silvano assieme a Diana, al contrario di ciò che è stato trovato in Pannonia. ${ }^{58}$ Nel territorio limitrofo l'associazione di Silvano ad Apollo, proviene solo da un'iscrizione rinvenuta a Savaria nella Pannonia Superiore, ${ }^{59}$ e da un rilievo votivo proveniente dalla Germania Superiore. ${ }^{60}$ Si potrebbe concludere che, nella Mesia Superiore e nelle province circostanti la dedica di Kalište è probabilmente l'unica in cui Silvano appare insieme a Diana e Apollo ed un'altra divinità.

Sul rilievo murato di Smederevo (cat. 9) Silvano è rappresentato insieme a due divinità. Questo rilievo è l'unica rappresentazione di Silvano, nella Mesia Superiore pervenuta ai nostri giorni. Oltre a questo, l'unico esem-

${ }^{39}$ SkovgaArd Jensen 1962, 11 sq.

${ }^{40}$ BEARD-NoRTH-PRICE 1998, 352.

${ }^{41}$ Silvano è invocato inseme alla Triade Capitolina nella Pannonia Superiore (1), assieme a Giove Ottimo Massimo, nella Pannonia Inferiore (3), nella Dacia (2), un'unica volta nella Pannonia Superiore, nella Mesia Superiore e nella Dalmazia, secondo EDH, http://edh-www.adw.uni-heidelberg.de/, (consultato 3.6.2016).

${ }^{42}$ Certi studi, per esempio quello di FARKAS $(1972,97)$, presumono che nella Pannonia il culto di Silvano faccia parte della religione ufficiale. Studi recenti invece lo negano, PERINIĆ MuRATOvić 2009, 292, 295; PERINIĆ Muratović-Vulić 2009, 172.

${ }^{43}$ Perinić Muratović 2009, 236.

${ }^{44}$ ILJug 1062; TitAq 305; TitAq 306; TitAq 342. È importante notare che le dediche a Silvano Augustus diventano più numerose proprio dal periodo dell'inizio della conquista romana della Pannonia, Perinić Muratović-Vulić 2009, 172.

${ }^{45}$ Dediche a Silvanus Augustus Sacrum: CIL III, 1146; IDR 3, 3, 405, fig. 296

${ }^{46}$ Dorcey 1992, 30
${ }^{47}$ Dedica a Silvanus Conservator da Salona: AEp 1997,

${ }^{48}$ AE 2001, 1664

${ }^{49}$ Birley 1978, 2535; Per le dediche dalla Mesia Superiore a Giove (IMS II, 2324), a Nettuno( IMS II 38) ed a Ercole si veda (IMS VI, 5).

${ }^{50}$ Giove con 1'appellativo Conservator: IMS II, 22; IMS

II, 23.

${ }^{51}$ Nettuno con 1'appellativo Conservator: IMS II, 38.

52 Mirković-DušAnić 1976, n. 108

${ }^{53}$ Per diverse interpretazioni delle divinità nell'iscrizione si veda MiRKOVIĆ 1986, 197.

${ }^{54}$ Dediche a Silvano e Diana: ILJug 760 e CIL III, 8483; Dedica a Silvano, Diana e Mercurio: ILJug 261.

${ }^{55}$ AEp 1939, 0241; ILBulg 197, T. 37, 197; ILBulg 208.

${ }^{56}$ AEp 1913, 54; CIL III, 7775.

${ }^{57}$ Aquincum: CIL III, 13368.

${ }^{58}$ Perinić Muratović-Vulić 2009, 170.

${ }^{59}$ AEp 1961, 2.

${ }^{60}$ CSIR Deutschland II, 4, n. 63. 


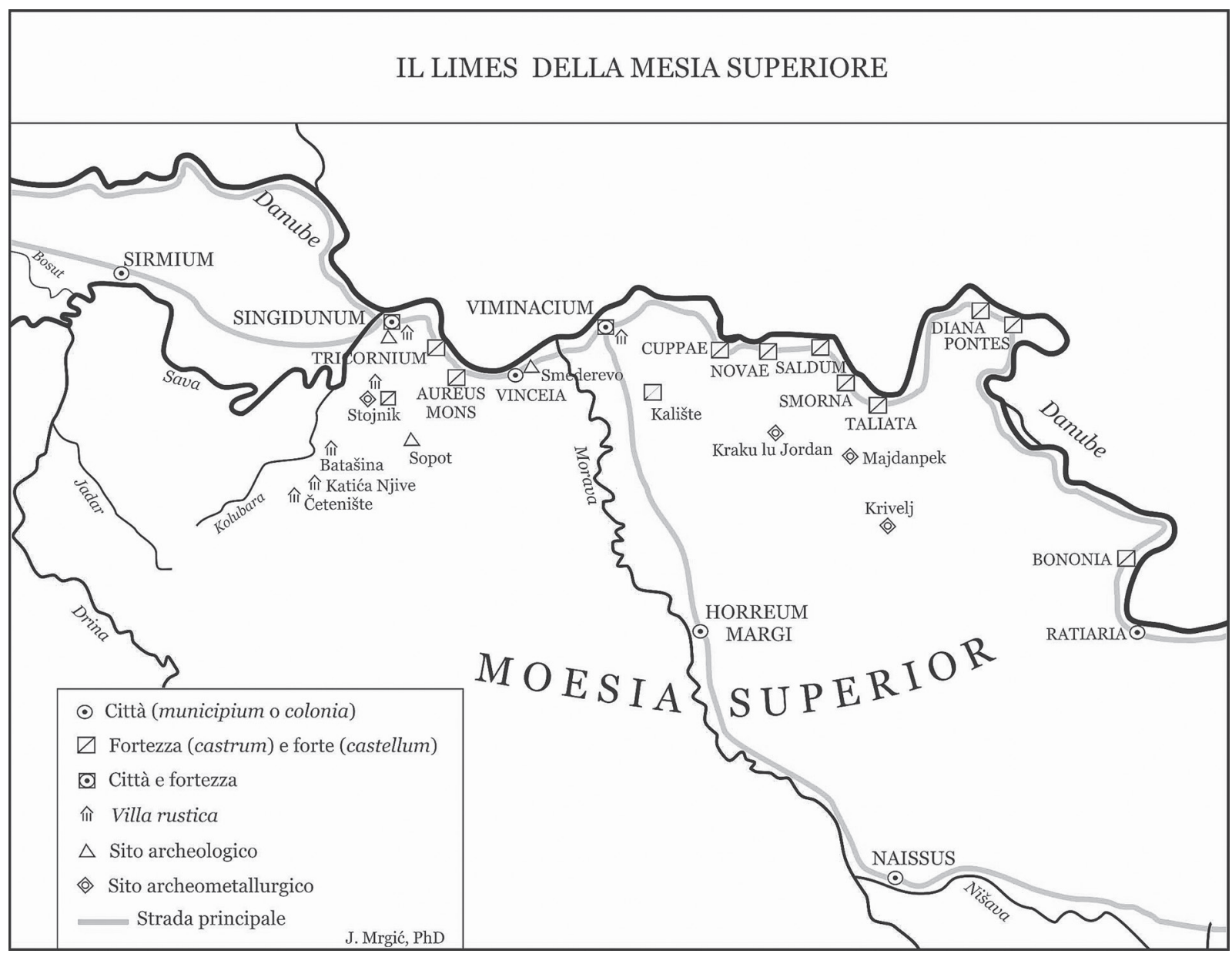

La mappa del limes della Mesia Superiore

pio che si potrebbe citare in materia, è un rilievo oggi ormai irreperibile, di provenienza sconosciuta, che prima della seconda guerra mondiale era conservato nel Museo Nazionale di Belgrado con Pan e le Ninfe. ${ }^{61}$ Il rilievo, proveniente da Smederevo è stato pubblicato nel 1903 da Vulić, Ladek e Premerstain, che ne hanno dato la seguente descrizione: "Tre figure in piedi, una accanto all'altra; nel centro si trova Giove, che nella mano sinistra regge lo scettro. Accanto ad esso sta probabilmente un'aquila. Sul lato sinistro si vede Silvano che regge una falce e un ramo con foglie. Sul lato destro di Giove, Marte regge lo scudo." ${ }^{62}$ Gli studiosi hanno datato il rilevo alla seconda metà del II secolo.

Innanzi tutto, è necessario chiarire che il rilievo è murato nella fortezza di Smederevo in un posto abbastanza inaccessibile per cui è difficilmente visibile in modo adeguato. Il rilievo, come già detto, rappresenta tre figure. Dal punto di vista del nostro studio, la figura più importante è quella del lato sinistro. Si tratta di un Silvano dall'aspetto antropomorfo, barbuto con berretto frigio, che veste una tunica con maniche, e per quanto si possa vedere, sulle gambe indossa stivali. Nella mano sinistra regge un ramo di pino e nella mano destra un coltello ricurvo, un pennato (falx alboraria, falx putatoria), lo strumento usato dai boscaioli per potare gli alberi. Non si vede bene se vicino al piede destro sia disteso un cane. Lo schema iconografico di Silvano sembra identico a quello di

${ }^{61}$ Il rilievo $(47 \times 42 \times 9 \mathrm{~cm})$ rappresentava Pan e le ninfe. Si vedevano le gambe di Pan ed una grande anfora e due ninfe vestite in tunica lunga (chitòn) e mantello (himàtion), che ballavano tenendosi per mano. Per il rilievo si veda Vulić1931, n. 619 con la foto. In possesso del Museo Nazionale di Belgrado c'era anche un frammento della parte inferiore (altezza 17,5 $\mathrm{cm}$ ) del rilievo con la raffigurazione di Pan e Menade, Baccante divina (Vulić 1931, n. 616). L'iconografia ha connotazione bacchica e connessioni con il mondo del vino.

${ }^{62}$ Vulić- LADEK- PREMERŠTAJN 1931, 46. 
un gruppo di rilievi provenienti dal limes della Pannonia Inferiore, gran parte rinvenuti ad Aquincum, con il cosiddetto tipo pannonico di Silvano. ${ }^{63}$ In questi rilievi, Silvano è rappresentato in tunica, con in testa un berretto frigio e con i soliti attributi: falcetto e ramo di pino. ${ }^{64} \mathrm{Il}$ falcetto, uno degli attrezzi agricoli più importanti $($ Ovid. Met. 14 , 628-631), che collega Silvano a Saturno, caratterizza Silvano come falx frondator (Ovid. Met. 14, 649) nel suo ruolo di inventore del plantatio. Il ramo di pino indica la natura selvaggia di Silvano che appartiene ai boschi, mentre il cane accanto a lui si potrebbe interpretare non solo come canis fidelis, ma anche come sumus custos (CEpigr 19, 2) ${ }^{65} \mathrm{Il}$ berretto frigio è un elemento tipico dell'iconografia legata alle divinità orientali, nello stesso tempo nella regione renana e danubiana, ${ }^{66}$ per Silvano è un simbolo dei lavori campestri. Lo schema iconografico pannonico di Silvano corrisponde alle raffigurazioni del Silvano italico, che, del resto, non è rappresentato troppo spesso. ${ }^{67} \mathrm{Il}$ tipo iconografico italico-pannonico di Silvano ci indicano che Silvano perde le sue caratteristiche rurali e cambia il suo campo di protezione qualificandosi più come protettore della casa e della famiglia in relazione con il fatto che molti dedicanti si spostavano per vivere nelle città. ${ }^{68}$

Rendić-Miočević formula l'ipotesi secondo la quale Domesticus e Silvester sono due appellativi che indicano due aspetti completamente diversi della divinità, visibili anche nell'iconografia ${ }^{69} \mathrm{Il}$ primo tipo iconografico è quello del Silvano antropomorfo cioè, quello italico-pannonico, mentre il secondo è teriomorfo, raffigurato frequentemente nella Dalmazia, spesso insieme a Diana e alle Ninfe con nomi italici (Silvanus, Diana, Nymhae).$^{70}$ Esistono due rilievi raffiguranti il Silvano pannonico, antropomorfo, con dediche a Silvanus Domesticus. Uno proviene da Aquincum (TitAq I, 320), dalla Pannonia Inferiore e l'altro da Brigetio, dalla Pannonia Superiore (RIU 2, 474). D'altra parte, sono stati rinvenuti rilievi con Silvano teriomorfo, uno proveniente da Aquincum con la dedica a Silvanus Silvester (CIL II, 3499), ${ }^{71}$ e l'altro da Aequinoctium, dalla Pannonia Superiore con la dedica alle divinità silvestri (CIL III, 4534). Questa tesi di due aspetti completamente diversi della divinità è stata elaborata nonostante l'esistenza degli esempi in cui Silvano Silvester aveva l'aspetto antropomorfo. Uno proviene da Cirpi, oggi Visegrád, sita nella Pannonia Superiore (AEp 1971, 223), e gli altri dalla Dacia e dalla Germania Superiore. ${ }^{72}$ Rendić-Miočević, citando l'esempio di Cirpi lo spiega come la fusione dei due tipi iconografici di Silvano. ${ }^{73}$ Nella letteratura questo tipo di Silvano antropomorfo, reggendo in mano una lancia o più lance che, qualche volta si trasformano in pedum, è denominato Silvano Illirico. ${ }^{74}$ Le lance nella mano potrebbero fare allusione al cacciatore, al soldato oppure al sovrano. ${ }^{75}$ Sulla base dell'iconografia sembra accettabile che nel rilievo derivante da Smederevo (cat. 9) fosse rappresentato il Silvano pannonico e si potrebbe aggiungere che si tratti di un Silvano domestico.

I dati finora esposti non sembrano sufficienti per ipotizzare chi fossero effettivamente le altre due figure maschili nel rilievo reperito a Smederevo (cat. 9). La figura centrale è nuda e nella mano sinistra regge lo scettro. Non si vede bene se la figura al lato destro del rilievo regga nella mano sinistra uno scudo oppure si tratta di un pezzo di mantello. Vulić presume che le due figure maschili siano Giove e Marte. ${ }^{76}$ Questa ipotesi sembra plausibile, ma non soddisfacente poiché la condizione della conservazione del rilievo la pone in dubbio. Nelle province circostanti si rappresentava Silvano insieme alle Ninfe e a Diana, ma raramente con altre divinità. Silvano tra Genio e Mercurio appare nel rilievo proveniente da Savaria, dalla Pannonia Superiore, Nel rilievo già citato, proveniente dalla Germania Superiore. ${ }^{77}$ è assieme a Diana e Apollo. In un rilievo appartenente al British Museum di Londra

${ }^{63}$ LIMC VII, s.v. Silvanus, n. 69-76, dei quali quattro provenivano da Aquincum (n. 69-72)

${ }^{64}$ Questo tipo di rappresentazione di Silvano vestito è più recente di quello, dove la divinità veste una pelle animale NAGY1994, 771.

${ }^{65}$ NAGY1994, 771.

${ }^{66}$ Nelle regioni renana e danubiana Silvano era rappresentato anche con le lance, l'attributo dei soldati e dei dominatori, NAGY 1994, 771 .

${ }^{67}$ Di solito Silvano italico o romano appariva sotto l'aspetto di una robusta figura di uomo barbuto, coronato di pino, vestito con pelle di capra o clamide e con la tunica, con coltello e rami di pino nelle mani, VERZÁR-BASS-CAMUFFO 2003, 127, fut. 129 con la bibliografia precedente.

${ }^{68}$ Perinić Muratović-Vulić 2009, 170.

${ }^{69}$ RendiĆ-MioČEVIĆ 1980, 113sqq.

${ }^{70}$ RENDić-MiočEvić 1980, 105-132; Rendić-MiočEvić 1989, 461-506; RENDIĆ-MiočEvić 2007a, 33-43; RENDIĆ-MiočEvić 2007b, 13-30; Perinić Muratović 2009, 298; Perinić MuratovićVulić 2009, 169-170.

${ }^{71}$ Erdélyi 1974, n. 172; RENDIĆ-MioČEVIĆ 1980, T. 1/2; TitAq I, 341; NAGY 2007, 142 n. 158

${ }^{72}$ Per ara di Cirpi, RENDIĆ-MiočEvić 1980, 114, 119. Per altre are votive provenienti dalla Dacia (da Apulum: CIL III, 1155 e da Napoca: AEp 1933, 20), e dalla Germania Superiore, vedi STEIDL 2008, 155, n. 152

${ }^{73}$ Riguardo alla fusione dei due tipi iconografici di Silvano, RENDIĆ-MiočEvić 1980, 114, 119.

74 Tipo di Silvano Illirico proveniente dalla Pannonia (n. 77-79), dalla Dacia (n. 80-89), e anche dalla Germania (n. 90-92), LIMC VII, s.v. Silvanus, 767.

${ }^{75}$ NAGY 1994, 772.

${ }^{76}$ Vulić-LADEK-PremerŠTAJN 1931, 46.

${ }^{77}$ CSIR, Deutschland II, 4, n. 63. 
erano raffigurati Giove, Pan e tre Ninfe delle acque stagnanti. ${ }^{78}$ Pertanto, se si dovesse stabilire che Silvano è associato a Giove e Marte, non si tratterebbe di un gruppo comune. Il rapporto tra Silvano e Marte è complesso, già da Cato (Agr. 83) è stata individuata una preghiera agricola a Marte Silvano ma non ci sono rappresentazioni, oppure dediche in cui appaiano insieme. ${ }^{79}$ D'altra parte, ci sono dediche a Silvano e Giove, specialmente nella Pannonia Inferiore e nella Dacia, e spunta alcuna, ma in minor numero, anche nella Mesia Inferiore e nella Dalmazia. ${ }^{80}$ Come si vede l'interpretazione del rilievo è molto complessa e comunque richiede più attenzione e approfondimento di quello che abbiamo a diposizione in questa sede. Si potrebbe solo ipotizzare che, accettando la lettura del rilievo proposta da Vulić - secondo la quale sul rilievo sono raffigurati Giove, Silvano e Marte - Silvano potrebbe essere definito non solo protettore della casa, della famiglia e della proprietà alla periferia di un villaggio, ma anche un nume dei confini (tutor finium), che sovrintende al confine che separa le foreste dal terreno messo a coltura, i pascoli dalle zone boschive, ma anche patrono delle frontiere dell'Impero. ${ }^{81}$

Non ci sono molti dati che ci permettono di comprendere chi erano le persone devote a Silvano nel limes della Mesia Superiore. La qualità inferiore delle are, gran parte in arenaria (cat. 3,8) e calcare (cat. 2, 6, 7) indica che si tratta di dedicanti non benestanti, forse di origini umili. Mancano anche i dati concernenti i devoti (cat. 8), oppure i nomi sono danneggiati o non si riesce a distinguerli con precisione (cat. 6). Hanno il gentilizio imperiale e i cognomina non abbastanza eloquenti per permettere di comprendere le loro particolari etnie (cat. 2 e 3 ). L'origine di M. Ulpius Terentius (cat. 3) è interpretata in modi diversi. Papazoglou ipotizza che sia originario da Teres nella Dardania ${ }^{82}$ mentre Mócsy presume che provenga dal territorio di Ulpiana, ${ }^{83}$ che pare di aver ottenuto lo status di municipium sotto l'imperatore Traiano all'inizio del II secolo. ${ }^{84}$ Dušanić ritiene che le origini di M. Ulpius Terentius risalissero proprio alla Mesia Superiore, oppure che si potrebbe collegare il suo cognomina con Teres, località della Dalmazia e soprattutto nella Dalmazia centrale. ${ }^{85}$ Čerškov cita due dediche a Silvano che provengono proprio da Ulpiana ${ }^{86}$ a favore dell'ipotesi della sua origine da Ulpiana, una è la dedica a Silvano Sacrum, fatta da un decurione e dai membri dell'ordine senatorio, ${ }^{87} \mathrm{e}$ l'altra è la dedica a Silvano Deus Sacrum, di un certo Rufus. ${ }^{88}$ D'altra parte le dediche a Silvano Augustus erano molto frequenti in Dalmazia. ${ }^{89}$ In mancanza di altri dati è difficile concludere quale fossero le vere origini di M. Ulpius Terentius, dedicante a Silvano e proveniente da Kosmaj.

Solo in due casi si può dire con certezza che i praticanti del culto provenissero dall'esercito romano. Una dedica da Kalište (cat. 7) fu eretta da Aelius Valentinus, duoviro (IIvir) della colonia, dignitario di Viminacium, membro della élite della città e l'altra dedica trovata a Kosmaj (cat. 5) fu eretta da un beneficiarius. Anche nella Dalmazia gran parte dei dedicanti faceva parte dell'esercito romano, mentre nella Pannonia la maggior parte di loro apparteneva alla popolazione civile, e solo un quarto apparteneva all'esercito romano. ${ }^{90}$

Nel limes della Mesia Superiore Silvano era venerato piuttosto in posti lontani dai centri delle città. Solo un'ara votiva, oggi perduta, è stata trovata nel castrum di Singidunum (cat. 1), mentre due dediche sono state rinvenute nel castellum sito vicino a Kalište (cat. 7-8), tre dediche nella zona della miniera della montagna di Kosmaj (cat. 3-5) e una alla periferia del territorio di Singidunum (cat. 2). Purtroppo, una dedica oggi irreperibile, rinvenuta a Smederevo (cat. 6) e il rilievo murato nella fortezza medievale di Smederevo (cat. 9) non possiedono un' esatta indicazione di provenienza. Questi reperti epigrafici ed archeologici risalgono ad un periodo che va dal I secolo fino alla fine del III secolo oppure, forse più precisamente, tra il II e III secolo.

Nella maggior parte dei casi Silvano è venerato come divinità italica (cat. 4, 6, 8, 9), grazie all'influenza derivante dalla Pannonia Inferiore che, veniva venerato con l'appellativo Domesticus (cat. 4, 6, 8), come una divi-

${ }^{78}$ LIMC VII, s.v. Zeus/Juppiter, n. 241.

${ }^{79}$ DORCEY 1992, 21, 120sqq.

${ }^{80}$ Pannonia Inferiore (AEp 1994, 1430; AE 1994, 1406; AEp 1966, 304), Dacia (AEp 1976, 576; CIL III, 890), Mesia Inferiore (AEp 1922, 70) e Dalmazia (ILJug 2003).

${ }^{81}$ Perinić Muratović-Vulić 2009, 178.

${ }^{82}$ Papazoglou 1969, 181.

${ }^{83}$ Mócsy 1970, 83-84.

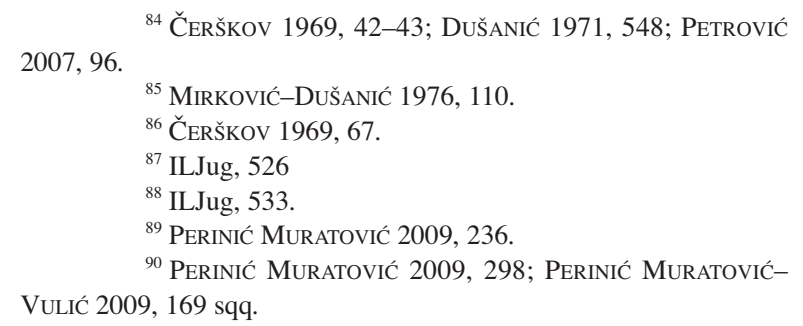


nità domestica che garantisce il benessere alle aziende agricole, ai pascoli e protegge la domus come protettore della casa e della famiglia. Si potrebbe presumere che Oppius Maximus avesse innalzato un'ara a Silvano Domesticus per proteggere sia il suo focolare domestico sia il terreno arabile in Kosmaj (cat. 4) e forse anche altre due are - una trovata a Kalište che. serviva a proteggere una proprietà agricola vicino al castellum (cat. 8) ed un'altra vicino a Smederevo (cat. 6) - erano state erette per lo stesso scopo. Nel rilievo proveniente da Smederevo Silvano aveva l'aspetto italico, cioè pannonico (cat. 9).

Nell'area suburbana di Singidunum (cat. 2) il culto del dio Silvano era diverso. Nella sua proprietà, Iulius Septumus ha eretto la dedica a Silvano Silvester, probabilmente per proteggere i suoi pascoli o il bestiame. Nelle vicinanze sono state trovate altre dediche alle divinità protettrici della fertilità, dell'agricoltura e della viticoltura, ${ }^{91}$ che, indicano l'esistenza di villae rusticae. La prima è una dedica a Giove, Terra Mater, Liber e Libera da parte di T. Aurelius Atticus, veterano della legione IIII Flavia. L'altra è una dedica a Liber e Libera (IMS I, 17). É difficile stabilire le origini di Iulius Septimus, un liberto imperiale, ma non si deve sottovalutare che il dedicante dell'altra ed unica dedica alle divinità silvestri proveniente dalla Mesia Superiore, da Viminacium (IMS II, 41), era una persona di condizione servile e di origine greca. La presenza del dio Silvano, cioè Silvester con aspetto teriomorfo è prevalente in Dalmazia, luogo in cui il carattere della divinità era Illirico, cioè indigeno. Risulta difficile concludere se anche alla periferia di Singidunum, Silvano fosse venerato come divnità con carattere indigeno, come interpretatio romana della una divinità locale.

Non sappiamo quale fosse il tipo dell'iscrizione dedicata al dio Silvano da M. Ulpius Tenerntius (cat. 3), cioè non ci risulta chiaramente se questa dedica proveniente dall'area mineraria di Kosmaj fosse di carattere locale. Il cognomen del devoto può indicare che le sue origini risalissero alla Mesia Superiore oppure alla Dalmazia. L'appellativo Augustus, già comune nella Dalmazia, potrebbe indicare l'ipotesi che il devoto volesse assicurargli un posto nel pantheon romano. É difficile verificare se Silvano in questa dedica avesse l'aspetto indigeno, illirico, di una divinità locale assimilata attraverso una interpretatio romana alla divinità italica.

Comunque pare che, i diversi caratteri di Silvano coesistessero contemporaneamente. Non abbiamo dati che ci permettano di identificare il primo aspetto della divinità apparsa come protettore dei boschi, dei pascoli, delle coltivazioni o della casa. E d'altronde non si possono distinguere nemmeno le zone geografiche secondo la prevalenza dell' aspetto con cui questa divinità vi era apparsa.

Le dimensioni modeste delle are indicano che si tratta di un culto di carattere privato, legato alla casa. La qualità delle pietre, tra le quali la maggior parte è arenaria (cat. 3, 8) e calcare (cat. 2,6,7) indica le origini umili dei dedicanti. Solo due iscrizioni rivelano le loro professioni e la loro provenienza dall'esercito romano: una da Aelius Valentinus (cat. 7), duoviro della colonia, dignitario di Viminacium e l'altra da un beneficiarius (cat. 5).

Si potrebbe concludere che nella devozione di Silvano nel limes della Mesia Superiore prevalesse, sotto gli influssi provenienti dalla Pannonia Inferiore l'aspetto italico (cat. 4, 6, 8, 9) e domestico (cat. 4, 6, 8). Purtroppo, in base al materiale epigrafico e archeologico oggi a nostra disposizione risulta, difficile avanzare conclusioni più precise. Per questo motivo si spera che nuove indagini archeologiche future contribuiranno a una più completa comprensione del culto di Silvano in questo territorio.

BIBLIOGRAFIA

BEARD-NORTH-PRICE 1998

BEKAVAC 2011

BIRLEY 1978

BREIN 1966-1967

CEpigr

ČERŠKOV 1969

Clauss 1988

CSIR Deutschland II, 4

\footnotetext{
= M. BEARD-J. NoRTH-S. Price: Religion of Rome 1: A History. Cambridge 1998.

$=$ S. BEKAVAC: Silvanus in Salona. VHAD 104 (2011) 151-166.

= E. BIRLEY: The religion of the Roman army. ANRW 2/16. Berlin-New York 1978, 1506-1541.

= F. BREIN: Eine unpublizierte Ara aus Kalište (Municipium) in Serbien. JÖAI 48 (1966-1967) 79-86.

F. BUCHELER-E. LOMMATZSCH (edd.): Carmina Latina Epigraphica. Lepizig 1926.

= E. ČERŠKOv: Rimljani na Kosovu i Metohiji [I Romani in Kosovo e Metochia]. Beograd 1969.

= M. Clauss: Omnipotens Mithras. Epigraphica 50 (1988) 151-161.

= Corpus Signorum Imperii Romani. II: Germania superior. 4: H. G. Frenz: Denkmäler römischen Götterkultes aus Mainz und Umgebung. Bonn 1992.
}

\footnotetext{
${ }^{91}$ Pilipović 2011, 71, 87.
} 
Cvetković 2011

Cvetković 2009

DIMITRIJEVIĆ 1996

DORCEY 1992

DUŠANIĆ 1971

DUŠANIĆ 2004

DZINO 2012

\section{EDH}

ERDÉLYI 1974

FARKAS 1972

FITZ 1993

HIRSCHFELD-SCHNEIDER 1885

IDR

ILBulg

ILIĆ 2012

ILJug

IMS

JOVANOVIĆ 2000
DOMASZEWSKI 1902

= B. CvetKović: Antičke spolije na zidinama smederevskog zamka i grada [Spolia antichi nella fortezza e la mura di Smederevo]. In: Pad Srpske despotovine 1459. godine. Ed.: M. Spremić. Beograd 2011, 393-407.

= S. CvetKović: Antička plastika Smederevske tvrđave - pregled dosadašnjih istraživanja [Scultura antica nella fortezza di Smederevo - una revisione delle ricerche precedenti]. Smederevski zbornik 2 (2009) 29-44.

= D. DimitriJević: Die Häfen der Classis Flavia Pannonica des Donausektors von Basiane (Taurunum - Cusum). In: Roman Limes on the Middle and Lower Danube. Ed.: P. Petrović. Belgrade 1996, $143-157$.

= A. VON DOMASZEWSKI: Silvanus auf lateinischen Inschriften. Philologus 61 (1902) 1-25.

= P. F. DoRCEY: The Cult of Silvanus: a study in Roman folk religion. New York 1992.

$=$ S. DUŠANIĆ: Heteroklitičko metalli u natpisima rudničkog novca [Eteroclitico metalli nelle iscrizioni sulle monete delle miniere]. ŽAnt 21/2 (1971) 535-554.

$=\mathrm{S}$. DUŠANIĆ: Roman mines in Illyricum: historical aspects. In: Dall'Adriatico al Danubio: L'Illirico nell'età greca e romana. Atti del convegno internazionale, Cividale del Friuli, 25-27 settembre 2003. Ed.: G. Urso. Pisa 2004, 247-270.

= D. DzINO: The cult of Silvanus: rethinking provincial identities in Roman Dalmatia. VAMZ 45 (2015) 261-279.

= Epigraphic Database Heidelberg (http://edh-www.adw.uni-rg.de/home/)

= G. ERDÉLYI: A római kőfaragás és kőszobrászat Magyarországon. Apollo könyvtár 5. Budapest 1974.

= Z. FARKAS: Notes sur le culte Scarbantien de Silvanus Augustus. ACD 8 (1972) 95-100.

= J. FITZ: Die Verwaltung Pannoniens in der Römerzeit. II. Budapest 1993.

= O. HIRSCHFELD-R. SCHNEIDER: Bericht über eine Reise in Dalmatien. AEM 9 (1885) 34-47.

= I. I. Russu et al.: Inscriptiile Daciei Romane (Dacia Superior) III/1-III/4. Bucharest 1977-1988.

= B. GEROV: Inscriptiones Latinae in Bulgaria repertae. Sofia 1989.

= O. ILIĆ: Poljoprivedna proizvodnja u rimskim provincijama na tlu Srbije od I do prve polovine V veka. [La produzione agricola nelle provincie romane nel territorio della Serbia dal I secolo alla prima metà del V secolo, tesi di dottorato discussa alla Facoltà di filosofia a Belgrado, 2012, non pubblicata.]

= A. ŠAŠEL-J. ŠAŠEL: Inscriptiones Latinae quae in Iugoslavia inter annos MCMXL et inter annos MCMLX et inter annos MCMLX et MCMLXX et inter annos MCMII etMCMXL repertae et editae sunt. Ljubljana 1963, 1978, 1986.

= Inscripitons de la Mésie Supérieure I, II, III/2, IV, VI. Ed.: M. Mirković et al. Beograd 1976-1995.

= A. JovAnović: Bronzana statueta Silvana iz Sirmijuma [La statuetta in bronzo da Sirmium]. Zbornik Muzeja Srema 4 (2000) 19-25.

Jovanović-PoP-Lazić-Mrkobrad 1992 = A. Jovanović-P. PoP-LAZIĆ-D. Mrkobrad: Novi arheološki nalazi na južnim obroncima Kosmaja [Nuovi reperti archeologici dalle pendici meridionali della montagna di Kosmaj]. Glasnik Srpskog Arheološkog Društva 8 (1992) 135-143.

KONDIĆ 1963

MARIĆ 1933

MARIĆ 1955

MATIJAŠIĆ-TASSAUX 2000

MIRKOVIĆ 1968

MiRKOVIĆ 1986

MiRKOVIĆ-DuŠANIĆ 1976

MóCSy 1970

Mócsy 1974

NAGY 1994

NAGY 2007

PAPAZOGLOU 1969

Perinić Muratović 2009
= V. Kondić: Donji Grad - antičko naselje [Donji Grad - insediamento antico]. AP 5 (1963) 73.

= R. MARIĆ: Antički kultovi u našoj zemlji [I culti antichi nel nostro paese]. Beograd 1933.

$=$ R. MARIĆ: Iz numizmatičke zbirke Narodnog muzeja [Dalla collezione numismatica del Museo Nazionale]. Starinar 5-6 (1955) 349-355.

$=$ R. MATIJAŠIĆ-F. TASSAUX: Liber et Silvanus. In: Les cultes polythéistes dans l'Adriatique romaine. Actes de Colloque. Éd.: C. Delplace, F. Tassaux. Bordeaux 2000, 76-91.

= M. Mirković: Rimski gradovi na Dunavu i Gornjoj Meziji [Le città romane sul Danubio e nella Messia Superiore]. Beograd 1968.

= M. Mirković (ed.): Viminacium et Margum. IMS II. Beograd 1986.

= M. MiRković-S. DuŠAnić: Singidunum et le nord-ouest de la province. IMS I. Beograd 1976.

= A. Mócsy: Gesellschaft und Romanisation in der römischen Provinz Moesia Superior. Budapest 1970.

= A. Mócsy: Pannonia and Upper Moesia. A history of the Middle Danube. Provinces of the Roman Empire. London-Boston 1974.

= Á. M. NAGY: Silvanus. In: LIMC VII/1-2. Zürich-München 1994, 763-773.

= M. NAGY: Lapidárium. A Magyar Nemzeti Múzeum régészeti kiállításának vezetője: Római Kőtár [Lapidario. Guida della mostra archeologica el Museo Nazionale Ungherese]. Budapest 2007.

$=$ F. PAPAZOGLOU: Srednjobalkanska plemena u predrimsko doba [Tribù nei Balcani centrali in epoca preromana]. Beograd 1969.

= LJ. PERINIĆ Muratović: Podrijetlo i narav boga Silvana u rimskim provincijama Dalmaciji i Panoniji. [L'origine e la natura del Dio Silvano nelle province romane della Dalmazia e della Pannonia, tesi di dottorato discussa alla Facoltà di filosofia a Zagrabia, 2009.] 
Perinić Muratović-Vulić 2009

PETER 1909-1915

Petrović 1991

Petrović 2007

Petrović 2009

Petrović 2011

Plemić 2012

PILIPOVIĆ 2011

POPOVIĆ 2009

RENDIĆ-MiočEvić 2007a

RENDIĆ-MiočEVIĆ 2007b

RENDIĆ-MioČEvić 1980

RENDIĆ-MioČEVIĆ 1989

RIU 1

RIU 2

SKOVGAARD JENSEN 1962

STEIDL 2008

TitAq

VASILJEVIĆ 2008

VERZÁR-BASS-CAMUFFO 2003

VuLIĆ 1909a

Vulić 1909 b

VuLIĆ 1913

VULIĆ 1931

VULIĆ-LADEK-PREMERŠTAJN 1931

Zotović 1992

ŽIVANOVIĆ 2013
= Lj. Perinić Muratović-H. Vulić: Razmatranja o Silvanovom kultu u Panoniji povodom nalaza žrtvenika u Vinkovcima [Ricerche sul culto di Silvano nella Pannonia dopo le scoperte delle are votive a Vinkovci]. Prilozi 26 (2009) 165-180.

$=$ R. PETER: Silvanus. In: Ausführliches Lexikon der griechischen und römischen Mythologie. IV: Qu-S. Hrsg.: W. H. Roscher. Leipzig 1909-1915, 824-877.

= P. Petrović: Classis Flavia Moesica na Dunavu u Gornjoj Meziji (Classis Flavia Moesica on the Danube in Upper Moesia). Starinar 40-41 (1991) 207-216.

= V. Petrović: Dardanija u rimskim itinerarima [Dardania negli itinerari romani]. Beograd 2007.

= V. Petrović: Les documents écrits relatifs aux voies de communication en Mésie Supérieure. Classica et Christiana 4/2 (2009) 137-171.

= V. Petrović: Les voies de communication en Mésie supérieure. In: L’homme et son environnement dans le Sud-Est européen. Actes du Xe Congrès de l'Association internationale d'études du Sud-Est européen, Paris, 24-26 septembre 2009. Paris 2011, 404-414.

= B. Plemić: Interpretatio romana of the forest deities in the Central Balkan Area. Glasnik Srpskog Arheološkog Društva 28 (2012) 47-62

S. PILIPOvić: Kult Bahusa na centralnom Balkanu, I-IV vek [Il culto di Bacco nei Balcani centrali, I - IV secolo]. Beograd 2011.

= I. Popović: Relief decorated handles of ceramic paterae from Sirmium, Singidunum and Viminacium. Starinar 48 (2009) 119-134

= A. RENDić-MiočEvić: Sylvanus of the Delmatae and traces of cult syncretism with Liber Histria Antiqua 15 (2007) 33-43.

= A. RENDIĆ-MiočEvić: Relief fragments bearing portrayals of Silvanus and Diana from the Tršćenica site in Split field. VAHD 100 (2007) 13-30.

= D. RENDIĆ-MiočEvić: Neki ikonografski i onomastički aspekti Silvanove »panonsko-iliričke« kultne zajednice [Alcuni aspetti iconografici e onomastici di Silvano insieme con la divinità »pannonico-illirica«]. VAMZ 3. ser. 12-13 (1979-80) 105-123.

= D. Rendić-MiočEvić: Silvan i njegova kultna zajednica u mitologiji Ilira. Ikonografska studija o spomenicima s teritorija Delmata [Silvano insieme ad altra divinità nella mitologia degli Illiri. Studio iconografico dei monumenti dal territorio dei Dalmati]. In: Iliri i antički svijet, Split 1989, 461-506 (Ilirske predstave Silvana na kultnim slikama s područja Delmata). Red.: I. Mimica. GZM, N.s. 10. Sarajevo 1955, 5-40).

= L. BARKócZI-A. Mócsy (Hrsg.): Die römischen Inschriften Ungarns (RIU). I: Savaria, Scarbantia und die Limes-Strecke Ad Flexum-Arrabona. Budapest 1972.

= L. BARKóCZI-A. MócsY (Hrsg.): Die römischen Inschriften Ungarns (RIU). II: Salla, Mogentiana, Mursella, Brigetio. Budapest 1976

$=$ S. SkovgaARD Jensen: Silvanus and his cult. AnalDan 2 (1962) 11-40.

= B. STEIDL: Welterbe Limes - Roms Grenze am Main. Obernburg am Main 2008

P. Kovács-Á. Szabó (ed.): Tituli Aquincenses I-II. Budapest 2009, 2010.

= LJ. VASILJEVIĆ: Arheološki spomenici boga Silvana u provincijama na tlu Srbije [Silvano. Reperti archeologici nelle province romane in Serbia, tesi di master discussa alla Facoltà di filosofia a Belgrado, non pubblicata]. ADD

= M. VerzÁr-BASS-M. CAmufFo: Trieste - Raccolte dei Musei Civico di Storia Ed. arte e relievi del propileo. 1. Roma 2003

= N. Vulić: Antički spomenici naše zemlje [Gli antichi monumenti del nostro paese]. Spomenik. Srpska kraljevska akademija 47 (1909) 109-191.

= N. Vulić: Antike Denkmäler in Serbien. JÖAI 12 (1909) 147-204.

= N. VuLIĆ: Archäologische Funde im Jahre 1912. AA 3 (1913) 337-344.

$=$ N. Vulıć: Antički spomenici naše zemlje [Gli antichi monumenti del nostro paese]. Spomenik. Srpska kraljevska akademija 71 (1931) 4-259.

= N. Vulić-F. LadeK-A. F. Premerštajn: Antički spomenici u Srbiji [Gli antichi monumenti in Serbia]. Spomenik. Srpska kraljevska akademija 39 (1931) 43-84.

= R. Zotović: Dva bronzana predmeta iz antičke zbirke Narodnog muzeja u Užicu [Due oggetti in bronzo dalla collezione della antichità del Museo Nazionale di Užice]. Glasnik Srpskog Arheološkog Društva 8 (1992) 102-104

= J. ŽIVANOvIĆ: Proizvodnja, uvoz i distribucija žitarica u rimskom periodu na tlu provincije Gornje Mezije. [Produzione, importazione e distribuzione del grano nella Mesia Superiore, tesi di dottorato discussa alla Facoltà di filosofia a Belgrado, non pubblicata] 